\title{
Multi-objective Optimization Model of Forest Spatial Structure Based on Dynamic Multi-Group PSO Algorithm
}

\section{Yuxin Wu}

Central South University of Forestry and Technology https://orcid.org/0000-0001-6858-0855

Jianjun Li (D 540177328@qq.com )

Central South University of Forestry and Technology https://orcid.org/0000-0002-6484-4443

Jing Huang

Central South University of Forestry and Technology

Dongsheng Qing

Central South University Xiangya Stomatological Hospital

Wei Ai

Central South University Xiangya Stomatological Hospital

\section{Peng Chi}

Hunan University

\section{Keqin Li}

SUNY New Paltz

\section{Research Article}

Keywords: Dynamic multi-objective, Multi-group particle swarm optimization, Pareto optimal solution set, Spatial structure optimization, Tracking error

Posted Date: March 7th, 2022

DOI: https://doi.org/10.21203/rs.3.rs-1398671/v1

License: (1) (i) This work is licensed under a Creative Commons Attribution 4.0 International License. Read Full License 


\title{
Multi-objective Optimization Model of Forest Spatial Structure based on Dynamic Multi-Group PSO algorithm
}

Yuxin $\mathrm{Wu}^{\mathrm{a}}$, Jianjun $\mathrm{Li}^{\mathrm{a}}{ }^{*}$, Jing Huang ${ }^{\mathrm{a}}$, Dongsheng Qing ${ }^{\mathrm{a}}$, Wei $\mathrm{Ai}^{\mathrm{a}}$, Peng Chi ${ }^{\mathrm{b}}$, Keqin $\mathrm{Li}^{\mathrm{c}}$

${ }^{\text {a }}$ College of Computer and Information Engineering, Central South University of Forestry and Technology, Changsha 410004, China

${ }^{\mathrm{b}}$ College of Information Science and Engineering, Hunan University, Changsha 410004, China

${ }^{c}$ Department of Computer Science, State University of New York, New Paltz, New York 12561, USA

\begin{abstract}
The multi-objective optimization problem, as one of the most popular hotspots in the current research, is facing both a big opportunity and a great challenge. Multi-group particle swarm optimization is often used to solve multi-objective optimization problems, however, the multi-group particle swarm optimization algorithm is more commonly used in solving static multi-objective optimization problems and less frequently used in solving dynamic multi-objective optimization problems. In solving dynamic multi-objective optimization problems, the algorithm lacks the corresponding environment detection mechanism. In this work, Dynamic Multi-Group Particle Swarm Optimization Algorithm is proposed and verified to solve multi-objective optimization problems of forest spatial structure. The proposed algorithm introduces a new mechanism for environmental detection, which can sense the changes of the situated


environment and make the multi-objective optimization results more suitable for the dynamic actual situation. The results show that the average generation distance $(\overline{G D})$ of the proposed algorithm is less than 0.0753 , and the average metric of maximum spread $(\overline{M S})$ is greater than 0.9852 . The spatial structure of the target forest has been optimized three times. The mingling intensity, volume, neighborhood comparison, and open ratio are increased by $40.6 \%, 2.5 \%, 18.9 \%$, and $11.8 \%$, respectively; while the competition index and angle index are decreased by $37.9 \%$ and $13.1 \%$, respectively. Obviously, the various indicators of the forest improved by our scheme are better than those before optimization.

Keywords: Dynamic multi-objective; Multi-group particle swarm optimization; Pareto optimal solution set; Spatial structure optimization; Tracking error. 


\section{Introduction}

Multi-objective optimization problems have always been one of the key and difficult points in scientific and engineering research [1]. Most multi-objective optimization problems are time- or environment-dependent, and when the environment changes, the objectives, parameters, and constraints of these problems may change as the environment changes, such multi-objective optimization problems are called dynamic multi-objective optimization problems. Evolutionary algorithms and swarm intelligence algorithms are increasingly popular applied to the establishment and optimization of dynamic multi-objective models [2-3]. In recent years, with the deepening of research and continuous improvement of evolutionary algorithms, swarm intelligence algorithms, which consist of ant colony algorithm, particle swarm optimization algorithm and fish swarm algorithm, have emerged and are introduced gradually into the field of multi-objective optimization [4-5]. When solving dynamic multi-objective optimization problems, the main points focus on how to track and predict the Pareto optimal solution set and how to obtain a uniformly distributed Pareto frontier as the time-dependent parameters change [6-8].

Forest structure is composed of the various components within the forest and their relationships with each other. The primary task of forest structure optimization is to establish the target structure of management optimization, i.e., what kinds of forest do we want to cultivate [10-11]. The business goal of forest ecosystems is to increase the complexity of forest structures. In the decision-making process of forest structure optimization, an optimized management of different forest types aims at the most suitable function [12]. The study of the optimization model of forest structure has gone through from a single wood function optimization model to a multi-objective 
optimization model which combines wood with ecological functions [13]. At the same time, it has gone through from linear programming and target planning to nonlinear multi-objective optimization [14]. Meanwhile, the quantification of forest structure is mainly determined by four major indicators: tree species, diameter, height, and age. Obviously, there is a lack of quantitative expression of forest dynamic structure [15]. In addition, the multi-objective optimization technique has only focused on the application of static multi-objective optimization in forest structure space. It lacks the construction technique with spatial information and time information in the dynamic multi-objective optimization model of forest structure [16-17].

Modern information technology such as artificial intelligence and big data analysis in forest structure analysis has been extensively applied in the field of modern forest management [18]. In particular, the research on the restoration, reconstruction, and optimization of natural secondary forest structures with spatial information, requires intelligent processing methods to develop its dynamic structural laws and coupling functions [19]. Especially in recent years, there have been various solutions to dynamic multi-objective optimization problems. These solutions mainly contain multiple groups, group diversity, memory search strategies, and hybrid memory strategies. Research on group intelligent algorithm for solving dynamic multi-objective problems has been deepened greatly [20-23]. In the face of complex and variable multi-objective optimization of forest structures, particle swarm optimization is widely used to solve multi-objective optimization problems. This is mainly attributed to its simple concept, easy implementation, fast convergence, and few adjustment parameters [24-28].

Dynamic multi-objective optimization problems have been the focus of many scholars, especially in the field of engineering applications, many problems are timedependent and dynamic with the change of time, traditional methods are difficult to find 
the optimal solution of these problems, need to find a simple and convenient intelligent optimization algorithm to find the optimal solution. The forest spatial optimization problem involves many indicators, the forest itself grows with time and thus affects the forest structure, some indicators will be optimized with the change of forest structure, some indicators will become worse with the change of forest structure, and there are mutual constraints between these indicators, so the forest spatial structure optimization problem is also a dynamic multi-objective optimization problem. In addition, the swarm intelligence algorithm with fast convergence speed and high search accuracy is highly favored in solving multi-objective optimization problems. In this study, the actual constraints of forest spatial structure are improved accordingly in order to solve the optimal solution of forest spatial structure and propose corresponding stand management measures based on the output results, so as to improve the forest stand structure.

In this paper, dynamic multi-group particle swarm optimization algorithm, which introduces a new mechanism for environmental detection, is proposed and used to analyze the dynamic process of forest structure optimization. Also, the planning data of natural secondary forest examples is used to establish the dynamic optimization model of forest structure. The results will provide a new solution to the dynamic multiobjective optimization of forest structure.

\section{Related Work}

The particle swarm optimization algorithm is a basic method for optimizing multiple targets. However, it is easy to fall into local optimization, reduce population diversity, poorly handle discrete problems, and have low reliability. In response to these problems, Carlos A. Coello Coello et al. [29] proposed a classic multi-objective particle swarm 
algorithm. This algorithm utilizes an external archive controller to determine and store the non-inferior solution members of each generation of particles, and use it guide other particles to fly. In order to maintain the diversity of particles and improve the global search ability of particles, Zhengjia Wu et al. [30] proposed an adaptive particle swarm optimization algorithm for adjusting inertia weight and social acceleration coefficient. Aiming at the defect that particle swarm optimization algorithm is easy to fall into local optimum, Suresh K et al. [31] adopted an adaptive strategy to improve the algorithm's global search capability. In order to solve the multi-objective flexible workshop scheduling problem, Zhang Guohui et al. [32] proposed a hybrid particle swarm optimization algorithm. This algorithm combines the advantages of the particle swarm optimization algorithm and the tabu search method. Liu Y et al. introduced a central particle based on Linearly decreasing weight PSO (LDWPSO) and updated it as the swarm center in each iteration. All particles oscillate around the particle swarm center and gradually converge toward it. Therefore, it has more opportunities to guide the search of the whole swarm and achieves faster convergence[35]. Hakli $\mathrm{H}$ et al.[36] combined PSO with Levy flights to eliminate local minima and improve global search capability. Hemlata $S$ et al[37], introduced a multi-community particle swarm algorithm in solving multi-objective optimization problems to achieve better optimization performance. In the process of multi-objective optimization, these algorithms have improved the performance of particle swarm optimization algorithm to a certain extent.

However, most of these optimization algorithms are single-group optimization algorithms. When faced with complex practical problems, single-group particle swarm optimization algorithms become inapplicable. In solving the problem of changes in forest structure, the dynamic multi-group particle swarm optimization algorithm proposed in this paper can adapt to complex and changing environmental factors. At 
the same time, this algorithm not only can improve the population diversity and the algorithm adaptability, but also can effectively solve the dynamic multi-objective optimization problems. We believe that the improved algorithm proposed in this paper can also be applied to other fields of studies.

\section{Multi-objective Optimization Model of Forest Spatial Structure}

The Longhushan Forest Farm in Minjiang, Hunan Province, is located in Chishan Island, South Dongting Lake, and belongs to the evergreen deciduous zone of Central Asia. Typical forest, includes highland mixed forest, green oak, eucalyptus mixed forest, and salix triandroides community. A representative plot of broad-leaved forest community $(30 \mathrm{~m} \times 30 \mathrm{~m})$, is set up in a typical section. The selected plot is a natural secondary forest. The soil in this plot is a typical red soil type mainly consisting of five species, i.e., Quercus fabri Hance, thick tree, Camphor tree, and Castanopsis sclerophylla. The number of investigated plants is 153 . For the trees with a DBH greater than $5 \mathrm{~cm}$ in the plot, they are regarded as the representative trees and numbered. Here, all the adjacent trees in the number and the nearest neighboring wood in the surrounding area are investigated. The test content includes the tree species, $D B H$, tree height, plane coordinates, and crown width of each tree (object tree).

The optimization of forest spatial structure is a dynamic process. In this paper, the selected forest samples include four species of white, bitter, eucalyptus and thick trees, and the single tree growth model of each tree species is determined.

The breast diameter model and the tree height model of Quercus fabri are expressed as

$$
D B H=\frac{36.3286}{\left(1-1.0342 \times e^{-0.039133 \times t}\right) \frac{1}{-0.626634}}
$$




$$
H=\frac{24.7636}{\left(\left(1-0.472312 \times e^{-0.085921 \times t}\right)^{-0.163019}\right)}
$$

The breast diameter model and the tree height model of Castanopsis sclerophylla are

$$
\begin{gathered}
D B H=36.327 \times\left(1-e^{-0.029 \times t}\right)^{1.375} \\
H=21.257 \times e^{-2.406 \times e^{-0.066 t}}
\end{gathered}
$$

The breast diameter model and the tree height model of Cinnamomum camphora are

$$
\begin{aligned}
& D B H=29.4350 \times\left(1-e^{-\left(\frac{t-6.7868}{6.5338}\right)^{0.7221}}\right) \\
& H=\frac{10.0147}{\left(\left(1+3.7636 \times e^{-0.169394 \times t}\right)^{\frac{1}{0.930456}}\right)}
\end{aligned}
$$

The breast diameter model and the tree height model of Quercus myrsinae are

$$
\begin{gathered}
D B H=\frac{29.023}{\left(1-0.967 \times e^{-0.03826 \times t}\right)^{-0.59122}} \\
H=\frac{14.7526}{\left((1+6.619) \times e^{-0.10544 \times t}\right)^{\frac{1}{0.73387}}}
\end{gathered}
$$

where $D B H$ is the breast diameter of trees, $H$ is the height of the tree, and $t$ is the age of the tree.

Two goals of volume and competition index are directly related to the $D B H$ of the forest. Their dynamics are directly reflected in the $D B H$ model. Its dynamic target is denoted as

$$
\begin{aligned}
V_{i}(t) & =V_{m}\left(\frac{D B H_{i}(t)}{d_{m}}\right)^{b} \\
C I_{i}(t) & =\frac{1}{n} \sum_{j=2}^{n} \frac{D B H_{j}(t) L_{D}}{D B H_{i}(t) \cdot L_{i j}}
\end{aligned}
$$

where $V_{m}$ is a constant determined by the diameter and ground level of the forest, $d_{m}$ is the breast diameter of the reference wood $m$, and $L_{i j}$ is the distance between the reference wood $i$ and the adjacent wood $j$.

Since the neighborhood comparison and the open ratio change with time, the dynamic target is represented as

$$
N_{i}(t)=\frac{1}{n} \sum_{j=1}^{n} k_{i j}(t)
$$




$$
O P_{i}(t)=\frac{1}{n} \sum_{j=1}^{n} t_{i j}(t)
$$

when the reference tree $i$ is younger (or older) than the adjacent wood $j, k_{i j}(t)=0$ (or $\left.k_{i j}(t)=1\right)$; when the horizontal distance between the reference wood $i$ and the adjacent wood $j$ is greater (or less) than the difference between the heights of them, $t_{i j}(t)=1$ (or $\left.t_{i j}(t)=0\right)$.

As the age of the stand increases, new species may appear in the stand, while existing tree species may disappear due to the superiority of the dominant tree species. Some trees may die for various reasons. These factors will change the mingling intensity and angle index. These dynamic targets are expressed as

$$
\begin{aligned}
M_{i}(t) & =\frac{1}{n} \sum_{j=1}^{n} v_{i j}(t) \\
A_{i}(t) & =\frac{1}{n} \sum_{j=1}^{n} z_{i j}(t)
\end{aligned}
$$

when the reference wood $i$ and adjacent wood $j$ are the same (or different) type of tree, $v_{i j}(t)=0\left(\right.$ or $\left.v_{i j}(t)=1\right)$; when the angle formed by the reference wood $i$ and the adjacent wood $j$ is less (or larger) than the standard angle, $z_{i j}(t)=1$ (or $\left.z_{i j}(t)=0\right)$.

The overall objectives of the dynamic multi-objective optimization model for forest spatial structure are: While maintaining species diversity, the competition between forests is weakened, and the forests tend to be evenly distributed in space. If so, the overall structure of the forest tends to be healthy and stable. Therefore, the above six targets are selected as the sub-targets of the model. The model output is the target tree that needs to be adjusted. The optimized mathematical model can be described as

$$
\min F_{i}(t)=\left(M_{i}(t),-C I_{i}(t),-A_{i}(t), N_{i}(t), O P_{i}(t), V_{i}(t)\right)
$$




\section{Dynamic Multi-Group Particle Swarm Optimization Algorithm}

\subsection{Multi-Group Particle Swarm Optimization Algorithm}

The standard particle swarm optimization algorithm will use the following formulas when updating the particle velocity and position.

$$
\begin{gathered}
v_{t+1}=u v_{t}+c_{1} r_{1}\left(p \text { Best }_{t}-x_{t}\right)+c_{2} r_{2}\left(g \text { Best }_{t}-x_{t}\right) \\
x_{t+1}=x_{t}+v_{t+1}
\end{gathered}
$$

where $w$ is the inertia weight, $r_{1}$ and $r_{2}$ are random numbers between $[0,1], c_{1}$ and $c_{2}$ are learning factors, and $t$ is the current number of iterations. In order to prevent blind search of particles, the search range of particles will be given a certain limit. In other word, $v$ and $x$ are limited in a certain value range.

The Multi-Group particle swarm optimization algorithm is an algorithm based on multiple particle swarms. Here, the number of particle swarms is optimized according to certain rules during the search process. $N$ particles are randomly initialized in the definition domain, and these particles are equally divided into $M$ subgroups by the kmeans algorithm. In addition, select the particles with the shortest distance from other particles in the group as the central particle of the particle group in each subgroup. The core calculations are expressed as

$$
\begin{gathered}
x_{k}^{\text {Center }}=\arg \left(\min \sum_{j=1}^{\frac{N}{M}}\left\|x_{k}^{i}-x_{k}^{j}\right\|\right) ; i \in\left[1, \frac{N}{M}\right] \\
\operatorname{distance}\left(x_{k}^{i}, x_{k}^{j}\right)=\left\|x_{k}^{i}-x_{k}^{j}\right\|=\sqrt{\left(\sum_{l=1}^{n}\left(x_{k}^{i l}-x_{k}^{j l}\right)^{2}\right) / n}
\end{gathered}
$$

where $x_{k}^{\text {Center }}$ is the center position of each particle swarm, $\left(x_{k}^{i}, x_{k}^{j}\right)$ refers to the distance between particle $i$ and particle $j$, and $n$ denotes the dimension of the decision variable $x$. 
Specifically, after determining the central particles of the $M$ subgroups, the distance between the particle group $K$ and the other particle groups is obtained according to Eq. 19. Based on these obtained results, the dynamic particle group is constructed. Let the upper limit of the distance among particle groups be $D_{\max }$, the lower limit be $D_{\min }$, and the particle group with the smallest distance from the particle group $k$ be the particle group $a$. If distance $\left(x_{k}, x_{a}\right)>D_{\max }$, a new particle swarm $x_{m+1}$ is generated. If the minimum distance between a particle swarm and the other particle swarms is less than $D_{\text {min }}$, a particle swarm is deleted. The particle is calculated as

$$
x_{M+1}^{i l}=\left(x_{k}^{i l}+x_{a}^{i l}\right) / 2+c_{1}(-1)^{\text {round }\left(0.5+c_{2}\right)}\left|x_{k}^{i l}-x_{a}^{i l}\right| / 2
$$

where $x_{M+1}^{i l}$ represents the $l$-th dimension component of the $i$-th particle in the $(M+1)$ th particle group, $c_{1}, c_{2}$ are random numbers in $[0,1]$, and round( $\left.\cdot\right)$ denotes the rounding function.

In order to find the optimal solution set of multiple targets, it is necessary to update the particle's own position and speed

$$
\begin{aligned}
v_{k}^{i}(t+1)= & \omega v_{k}^{i}(t)+c_{1} r_{1}\left[\operatorname{pbest}_{k}^{i}(t)-x_{k}^{i}(t)\right]+c_{2} r_{2}\left[\operatorname{lbest}_{k}(t)-x_{k}^{i}(t)\right] \\
& +c_{3} r_{3}\left[\operatorname{gbest}(t)-x_{k}^{i}(t)\right]
\end{aligned}
$$

where the velocities of particle $i$ in particle swarm $k$ at time $t$ and $t+1$ are denoted as $v_{k}^{i}(t)$ and $v_{k}^{i}(t+1)$, respectively. Its optimal position of the particle at time $t$ is denoted as $\operatorname{pbest}_{k}^{i}(t) . x_{k}^{i}(t)$ represents the position of the particle $i$ at time $t$. The optimal position of the particle group of all particles in particle group $k$ at time $t$ is denoted as $\operatorname{lbest}_{k}(t)$. The global optimal position of all particles at time $t$ is $g b e s t(t)$. $c_{1}, c_{2}, c_{3}$ are constants which are used to adjust the effect of the particle itself, the particle group, and all particles on the particle $i . r_{1}, r_{2}, r_{3}$ are random numbers in [0,1]. $\omega$ represents the inertia factor whose value varies with the search process. It is also related to the current iteration algebra $I_{c} I_{c}$ and the maximum iteration algebra $I_{\text {max }}$. The transformation formula of $\omega$ is 


$$
\omega=\omega_{s}-\left(\omega_{s}-\omega_{e}\right)\left(\frac{I_{C}}{I_{\max }}\right)
$$

where $\omega_{s}$ is the initial inertia factor, $\omega_{e}$ is the final inertia factor, $I_{c}$ is the current number of iterations, and $I_{\max }$ is the maximum number of iterations. The particle position is updated as

$$
x_{k}^{i}(t+1)=x_{k}^{i}(t)+v_{k}^{i}(t+1)
$$

where $x_{k}^{i}(t+1)$ and $x_{k}^{i}(t)$ are the positions of the particles $i$ at the time $t+1$ and $t$, respectively.

When the position of the particle is updated according to Eq. 23, there is a probability that the position of the particle is out of the defined domain. Such a particle is called an out-of-boundary particle. When an out-of-boundary particle appears, the velocity $v_{k}^{i}(t+1)$ in Eq. 23 is multiplied by an adjustment factor $\beta(\beta \in(0,1))$ to pull the outof-boundary particle back into the domain. The new formula of position is calculated according to Eq. 24. If this particle is still out of bounds after adjustment, it is necessary to reduce $\beta$ according to Eq. 25 until the particle returns to the decision space. Eqs. (24)-(25) can rewritten as

$$
\begin{gathered}
x_{k}^{i}(t+1)=x_{k}^{i}(t)+\beta v_{k}^{i}(t+1) \\
\beta=1 /\left(\delta^{2}+1\right)
\end{gathered}
$$

where $\delta$ is the number of adjustments.

\subsection{Environment Detection Mechanism}

The environment detection mechanism is introduced based on the existing multigroup PSO algorithm. Therefore, the particles can feel the changes of the environment with time, making the optimization results more realistic. Due to the dynamic multiobjective particle swarm optimization algorithm of file intersection, a file maintenance strategy based on fast European crowded distance is adopted. It randomly selects $20 \%$ 
of the individuals in the current population, calculates the Euclidean distance difference between the new fitness value and the original fitness value, and then takes the average to detect the environmental change. The calculation is given a s follow:

$$
\varepsilon=\frac{\sum_{i=1}^{n} \sqrt{\sum_{j=1}^{m}\left(f\left(x^{j}, T\right)-f\left(x^{j}, T-1\right)\right)^{2}}}{n}
$$

where $\varepsilon$ denotes a detection operator. This operator is used to detect whether the environment has changed, or whether the current moment's problem actually changes compared with that in the previous moment. $i$ represents one of $20 \%$ of the individuals in the current randomly selected population. $n$ refers to the number of randomly selected individuals. When the fitness function $f$ is the objective function, $m$ represents the target dimension, which is determined by the number of objective functions. When the fitness function $f$ is denoted by other functions, $m$ represents the number of other functions. $T$ represents the current environment, and $T-1$ represents the previous environment. On the basis of the actual problem to be solved, it is necessary to select the fitness function and set the threshold $\theta$ according to the relationship between the fitness function and the environment variable. If the detection operator $\varepsilon$ is greater than $\theta$, it is judged that the environment has changed, and the algorithm responds accordingly. On the contrary, the environment has not changed and the algorithm continues to run the steps in the current environment.

After detecting the change of the environment, it is necessary to determine whether the new environment is similar to the previous environment via the environment recovery operation. The calculation method of environmental similarity is similar to that of environmental change detection. It is should be noted that the environmental similarity is strongly correlated to the Euclidean distance difference between the new fitness value of the $k$ points saved in a previous environment and the original fitness value. 


$$
\phi=\frac{\sum_{i=1}^{k} \sqrt{\sum_{i=1}^{m}\left(f\left(x^{i}, T\right)-f\left(x^{j}, T\right)\right)^{2}}}{k}
$$

where $k$ refers to the number of representative points saved in the $T$ - $t h$ environment, $m$ denotes the target dimension, and $T$ represents the new environment. After the threshold $\eta$ is set, if the result is below the threshold $\eta$, it is judged that the $T$-th environment is similar to the current new environment. The information provided by the $T$-th environment can be restored to the current external file set, enabling the algorithm to quickly adapt to the new environment. The improved processes of algorithm are list as follows:

\footnotetext{
Algorithm 1 Dynamic Multi-Group Particle Swarm Optimization

1. Randomly generate $N$ particle in the domain, and randomly generate the initial position and initial velocity of each particle in the feasible domain of the decision variable;

2. Use the K-means algorithm to divide the randomly generated particles into $M$ group of particles;

3. Calculate the distance among particles within a group according to Eq. (19). If the minimum distance between a subgroup and other subgroups is greater than $D_{\max }$, a new particle group $x_{M+1}$ is generated;

4. Initialize the particle outer set $p_{k}^{j}$, the group outer set $L_{k}$, and the outer set $G$ of the global optimal solution of all particles to the empty set;

5. Save the non-dominated solution to the external concentration and update the optimal position; 6. Use Eq. (26) to detect the environmental change. If $\varepsilon>\theta$, the algorithm runs to the next step, otherwise it runs the Step 8;

7. Update the fitness value of the individual in the population under the new environment, and update the particle external set, the group external set, and the global optimal solution external set $G$;
}

8. Update the velocity and position of the particle according to Eq. (21) and Eq. (23), and calculate the current fitness value. If the new position exceeds the bounds of the domain, the 
cross-boundary particles are processed according to Eq. (24) and Eq. (25);

9. Find the distances among particle groups and insert or delete the particle group. For the inserted new particle swarm, initialize its particles and generate a particle outer set and a group outer set;

10. Determine whether the maximum iteration algebra is reached. If so, output the global optimal solution external set $G$ of all particles and the corresponding fitness function value; Otherwise return to Step 5 and continue to find the optimal solution.

\subsection{Performance Testing of Improved Algorithm}

Test function and performance indicators, i.e., FDA1, FDA3, dMOP1 and dMOP2, are selected to test the performance of multi-group PSO algorithm for dynamic multiobjective optimization problems. The convergence and distribution of the algorithm are evaluated by three indicators: Generation Distance $(G D)$, metric of maximum spread $(M S)$, and Tracking Error $(T E)$. These four test functions are defined as follows:

Table 1

Test Functions

\begin{tabular}{|c|c|c|c|}
\hline $\begin{array}{l}\text { Test } \\
\text { function }\end{array}$ & Objective function & $\begin{array}{l}\text { Decision } \\
\text { variables }\end{array}$ & Type \\
\hline FDA1 & $\left\{\begin{array}{l}f_{1}(x, t)=x_{1} \\
f_{2}(x, t)=g \cdot\left(1-\sqrt{\frac{f_{1}}{g}}\right) \\
g=1+\sum_{j=2}^{n}\left(x_{j}-G(t)\right)^{2} \\
G(t)=\sin (0.5 \pi t), t=\frac{1}{n_{T}} \cdot \frac{\tau}{\tau_{T}}\end{array}\right.$ & $\begin{array}{l}{[0,1] \times[-1,1]^{n-1}} \\
n=20\end{array}$ & $\begin{array}{l}P O S_{t}^{*} \\
\text { change } \\
P O F_{t}^{*} \\
\text { don't } \\
\text { change }\end{array}$ \\
\hline
\end{tabular}


FDA3

$$
\left\{\begin{array}{l}
f_{1}(x, t)=\sum_{j=1}^{5} x_{j}^{10^{2 \sin (0.5 \pi t)}} \\
f_{2}(x, t)=g \cdot\left(1-\sqrt{\frac{f_{1}}{g}}\right) \\
g=1+G(t)+\sum_{j=6}^{n}\left(x_{j}-G(t)\right)^{2} \\
G(t)=|\sin (0.5 \pi t)|, t=\frac{1}{n_{T}} \cdot \frac{\tau}{\tau_{T}}
\end{array}\right.
$$

$$
[0,1]^{5} \times[-1,1]^{n-5} \quad \operatorname{POS}_{t}^{*}
$$

$$
n=30
$$

change

$\mathrm{POF}_{t}^{*}$

change

$[0,1] \times[-1,1]^{n-1} \quad \operatorname{POS}_{t}^{*}$

$n=20$

don't

change

$\mathrm{POF}_{t}^{*}$

change

dMOP2

$$
\left\{\begin{array}{l}
f_{1}(x, t)=x_{1} \\
f_{2}(x, t)=g \cdot\left(1-\left(\frac{f_{1}}{g}\right)^{H(t)}\right) \\
g=1+\sum_{j=2}^{n}\left(x_{j}-G(t)\right)^{2}, G(t)=\sin (0.5 \pi t) \\
H(t)=1.25+0.75 \sin (0.5 \pi t), t=\frac{1}{n_{T}} \cdot \frac{\tau}{\tau_{T}}
\end{array}\right.
$$

$n=20$

change

$\mathrm{POF}_{t}^{*}$

change

Convergence index $G D$ is the degree of convergence of $P F_{\text {known }}$ to $P F_{\text {true }}$. It is calculated by

$$
G D=\sqrt{\frac{1}{n_{P F}} \sum_{i=1}^{n_{P F}} d_{i}^{2}}
$$

where $n_{P F}$ is the number of optimal solutions, $d_{i}$ is the distance between the $i$-th solution in the optimal solution set $P F_{\text {known }}$ and the closest solution in the true optimal solution set $P F_{\text {true }}$.

Use the average generation distance $\overline{G D}$ to evaluate the convergence of the algorithm in dynamic environmental, i.e., 


$$
\overline{G D}=\frac{1}{T_{\text {run }}}\left(\sum_{i=1}^{T_{\text {run }}} \frac{1}{T} \sum_{t=1}^{T} G D(t)\right)
$$

where $T_{\text {run }}$ is the number of times which the algorithm runs, $T$ is the number of times the environment changes during runtime.

Metric of maximum spread $(M S)$ can be used to obtain the $P F_{k n o w n}$, and then the $P F_{\text {true }}$ is covered by $P F_{\text {known. }}$. In a changing environment, the average metric of maximum spread is used to evaluate the performance of the algorithm, which is given by

$$
\begin{gathered}
M S=\sqrt{\frac{1}{n_{o b j}} \sum_{i=1}^{n_{o b j}}\left[\left(\min \left(f_{i}^{\max }, F_{i}^{\max }\right)-\max \left(f_{i}^{\min }, F_{i}^{\min }\right)\right) /\left(F_{i}^{\max }-F_{i}^{\min }\right)\right]^{2}} \\
\overline{M S}=\frac{1}{T_{\text {run }}}\left(\sum_{i=1}^{T_{r u n}} \frac{1}{T} \sum_{t=1}^{T} M S(t)\right)
\end{gathered}
$$

where $f_{i}^{\text {max }}$ and $f_{i}^{\text {min }}$ are the maximum and minimum values of the $i$-th goal of all solutions in the optimal solution set $P F_{\text {known }}$, respectively. $F_{i}^{\text {max }}$ and $F_{i}^{\text {min }}$ are the maximum and minimum values of the $i$-th goal of all solutions in the optimal solution set $P F_{\text {true }}$, respectively.

Besides the convergence and distribution of the test algorithm, $T E$ is also used to test the tracking performance of this algorithm. In a dynamic environment, the average tracking error $\overline{T E}$ is used to evaluate the tracking performance of the algorithm, which is calculated as follows

$$
\begin{gathered}
T E=\frac{1}{n_{n}} \sum_{j=1}^{n_{n} \Sigma}\left(\sqrt{\sum_{k=2}^{\operatorname{dim} \sum}\left(x_{j, k}^{i}-x_{k}^{i *}\right)^{2}}\right) \\
\overline{T E}=\frac{1}{T_{\text {run }}}\left(\sum_{i=1}^{T_{\text {run }}} \frac{1}{T} \sum_{t=1}^{T} T E(t)\right)
\end{gathered}
$$

where $n_{n}$ is the number of undominated solutions before the environment changes, $x_{j, k}^{i}$ is the value of the $k$-th dimension of the $j$-th non-dominated solution when the 
environment changes, and $x_{k}^{i *}$ is the $k$-th dimension of the true value closest to the $j$ th non-dominated solution.

\section{Performance Evaluation}

\subsection{Performance Testing of Improved Algorithm}

In the simulation experiment, the parameter settings are referred to the experimental settings in the PDMIOA[33] and dDMS-MOPSO[34]. The population size is $N=100$, the dimension of each particle is $k=10$, and the acceleration factor is $c_{1}=c_{2}=1.49445$. For each dynamic multi-objective problem, the frequency $\tau_{T}$, and the amplitude $n_{T}$ of the environmental change are $(25,10)$ and $(25,5)$, respectively. Under each combined environment, the number of environmental changes is 50 , and the environmental detection threshold is $\theta=0.000001$. For each test question, each algorithm runs 100 times independently and then the average value is calculated.

Four typical functions were tested. After each algorithms were run 100 times independently, the average generation distance $\overline{G D}$ and average maximum coverage $\overline{M S}$ of each function under different environments were obtained.

Table 2

The mean values of GD and MS are obtained by three algorithms

\begin{tabular}{lcllllll}
\hline & & \multicolumn{3}{c}{$\overline{G D}$} & & & $\overline{M S}$ \\
\multirow{2}{*}{ Test } & & & & & & & \\
\cline { 3 - 8 } function & $\left(\tau_{T}, n_{T}\right)$ & PDMIOA & dDMS- & Improve & PDMI- & dDMS- & Improve \\
FDA1 & & & MOPSO & algorithm & OA & MOPSO & algorithm \\
& $(25,10)$ & 0.0039 & 0.0325 & 0.0026 & 0.9638 & 0.9546 & 0.9954 \\
& $(25,5)$ & 0.0071 & 0.0323 & 0.0039 & 0.9912 & 0.9892 & 0.9965
\end{tabular}




\begin{tabular}{lccccccc} 
FDA3 & $(25,10)$ & 0.0210 & 0.0815 & 0.0306 & 0.9698 & 0.9759 & 0.9915 \\
& $(25,5)$ & 0.4962 & 0.1006 & 0.0753 & 0.9805 & 0.9814 & 0.9961 \\
dMOP1 & $(25,10)$ & 0.0005 & 0.0018 & 0.0002 & 0.9897 & 0.9918 & 0.9852 \\
& $(25,5)$ & 0.0006 & 0.0010 & 0.0004 & 0.9746 & 0.9682 & 0.9904 \\
dMOP2 & $(25,10)$ & 0.0015 & 0.0362 & 0.0008 & 0.9865 & 0.9853 & 0.9916 \\
& $(25,5)$ & 0.0044 & 0.0315 & 0.0014 & 0.9927 & 0.9821 & 0.9931 \\
\hline average & & 0.0669 & 0.0397 & 0.0144 & 0.9811 & 0.9786 & 0.9925
\end{tabular}

Since the Pareto optimal solution set $\operatorname{POS}_{t}^{*}$ of the test function FDA1 changes and the Pareto optimal front end $P O F_{t}^{*}$ does not change, this problem belongs to the first type of dynamic multi-objective optimization problem. In the case of the same environmental change, the higher the frequency of environmental change, the smaller the tracking error, and the better the tracking performance of the algorithm. It is easy to understand the reason: the larger the frequency value of the environmental change, the more time the algorithm optimization. For a same frequency of environmental change, the smaller the variation range of the environment, the smaller the variation range of the obtained tracking error, and the more stable the algorithm.

Table 2 shows that the improved algorithm can get the minimum average generation distance $\overline{G D}$ and the largest average coverage $\overline{M S}$. In most cases, the improved algorithm possesses better performance when solving multi-objective optimization problems. Compared with the other two algorithms, through the test of the test functions FDA1, FDA3, dMOP1, dMOP2 (Fig.1 to Fig.4), the improved algorithm shows the best tracking performance except for the individual environments. Therefore, compared with the other two algorithms, the improved algorithm proposed in this paper has obvious improvement in convergence performance, distribution performance, and tracking performance. 


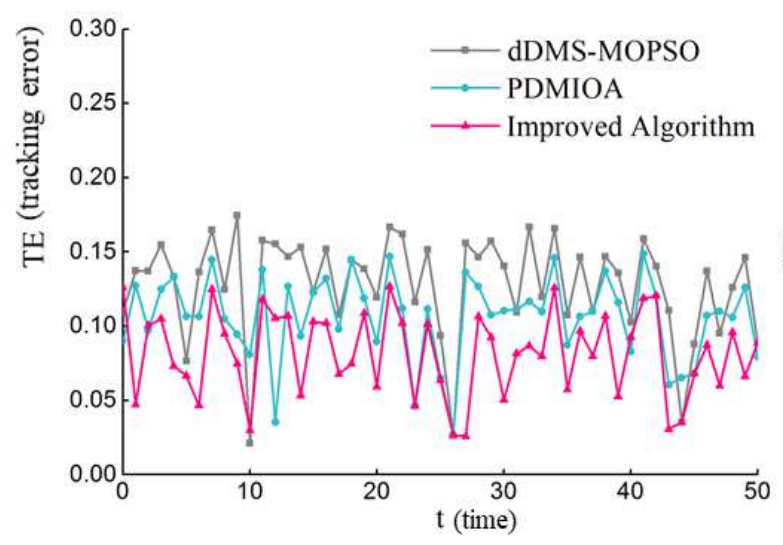

(a) $\tau_{\mathrm{T}}=25, \mathrm{n}_{\mathrm{T}}=10$

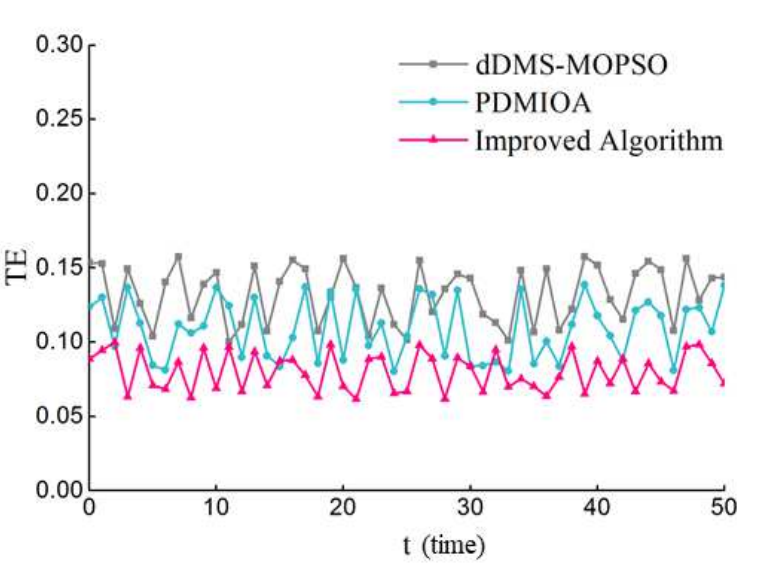

(b) $\tau_{\mathrm{T}}=25, \mathrm{n}_{\mathrm{T}}=5$

Fig. 1. Tracking error diagram under test function FDA1

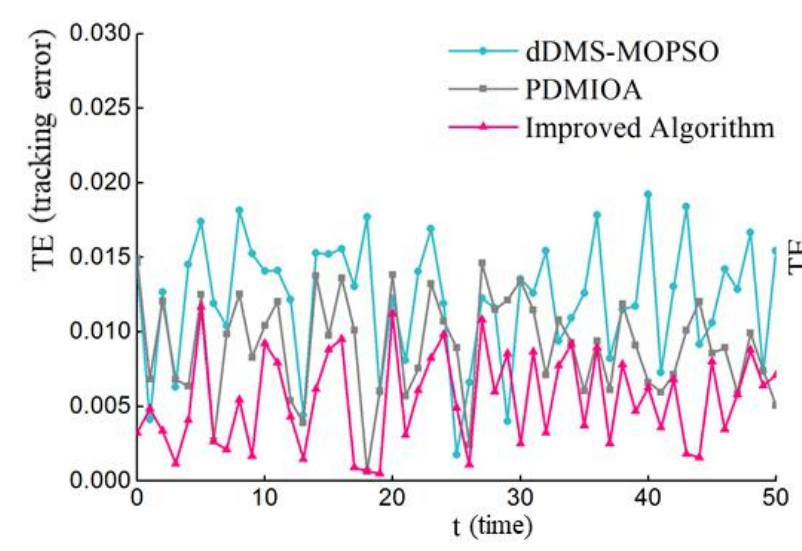

(a) $\tau_{\mathrm{T}}=25, \mathrm{n}_{\mathrm{T}}=10$

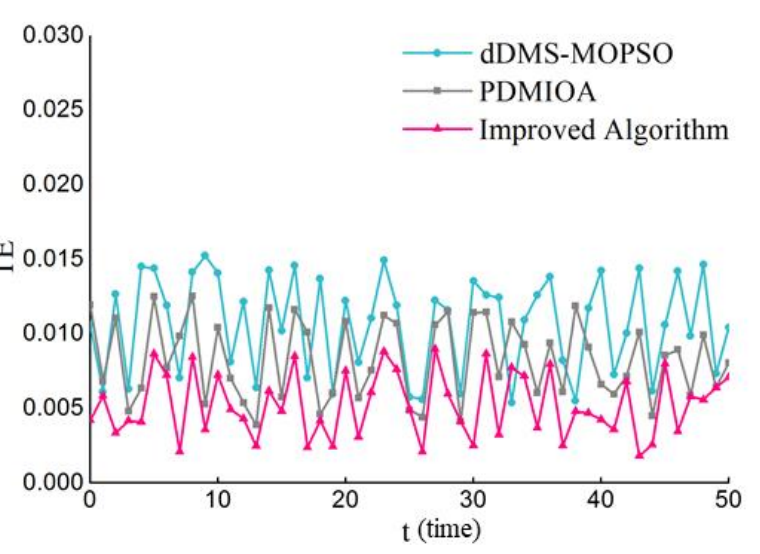

(b) $\tau_{\mathrm{T}}=25, \mathrm{n}_{\mathrm{T}}=5$

Fig. 2. Tracking error diagram under test function FDA3

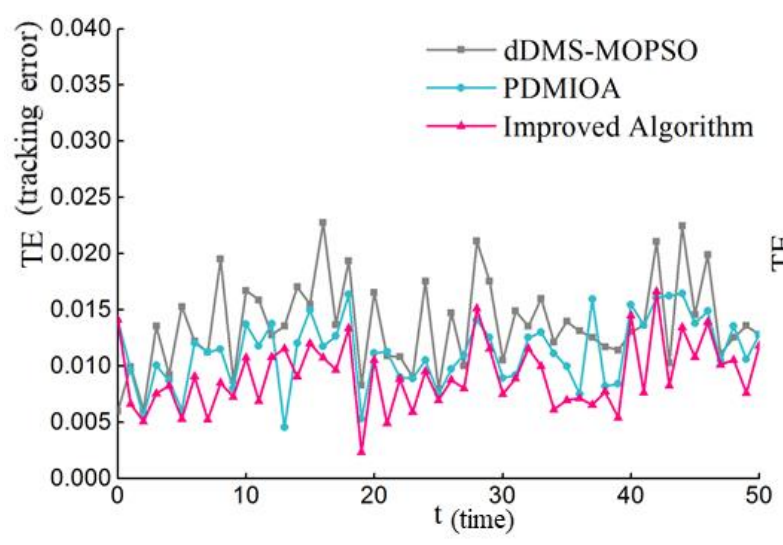

(a) $\tau_{\mathrm{T}}=25, \mathrm{n}_{\mathrm{T}}=10$

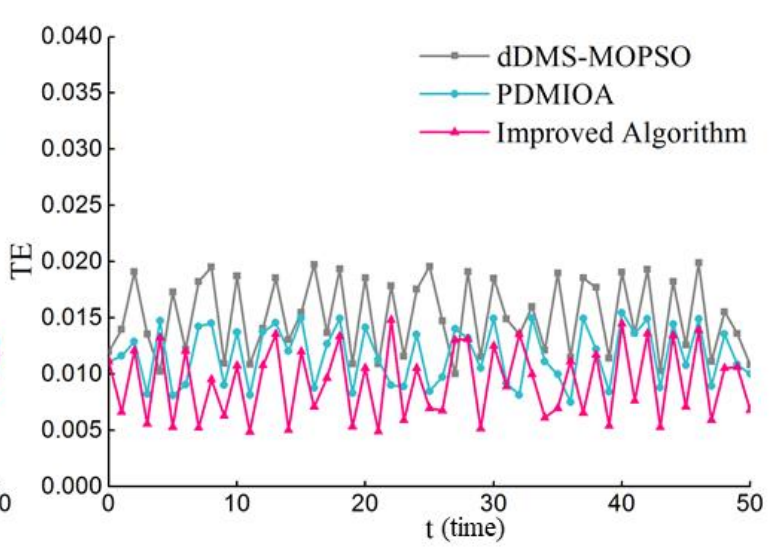

(b) $\tau_{\mathrm{T}}=25, \mathrm{n}_{\mathrm{T}}=5$

Fig.3. Tracking error diagram under test function dMOP1 


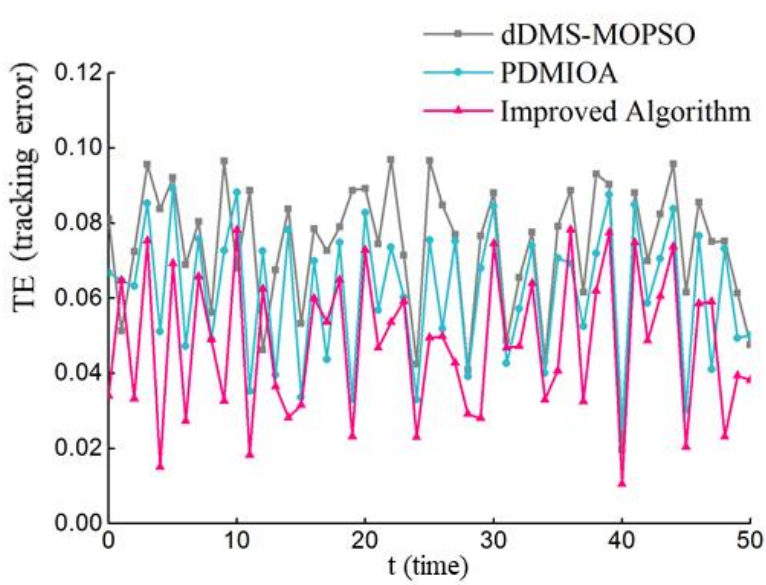

(a) $\tau_{\mathrm{T}}=25, \mathrm{n}_{\mathrm{T}}=10$

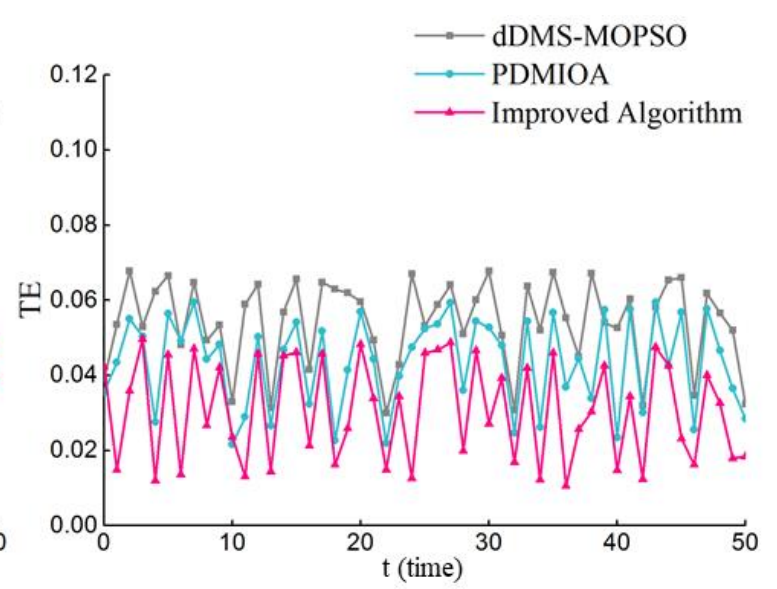

(b) $\tau_{\mathrm{T}}=25, \mathrm{n}_{\mathrm{T}}=5$

Fig. 4. Tracking error diagram under test function dMOP2

5.2 Test of Realistic Stand Model and Analysis of Simulation Optimization Effects

\subsubsection{Model Output and Structure Optimization}

Under the Windows 7 operating system, the experimental data is programmed by MATLAB 7.1. The experiment finally outputs the forest trees that need to be regulated in the forest (i.e. the target tree), and the output target tree is the key to affect the spatial structure of the forest. The resulting data is combined with the original data and plotted using the OriginPro 8 software. The position information of the particles represents the position information of the trees in this forest, so the search dimension of the particle is 2 . Since the coordinates of the position after normalization are all in the range of $[0$, 1], the position of the particle is in the range [0,1], i.e., $x_{\max }=1, x_{\min }=0$. Set the maximum speed of the particle $v_{\max }=x_{\max }-x_{\min }, v_{\min }=-v_{\max }$. In natural management, the amount of harvesting usually does not exceed $15 \%$ of the total number of trees in the forest. Therefore, in this study, the size of the particle group $(N)$ is set to $15 \%$ of the total number of trees, i.e., $N=20$. All particles are divided into 4 subgroups, i.e., $m=4$. Therefore, the number of particles per population is 5. According to the 
adjustable frequency of the forest structure, three environmental variables are set at three intervals, $T h=1$ (acquisition data time), $T h=2, T h=3$.

The results of the model output under three environmental variables in the first experiment are shown in Fig.5, Fig.6, and Fig.7, representing the spatial distribution of the target tree when $T h=1, T h=2$, and $T h=3$, respectively. The specific parameter settings are shown in Table 3.

Table 3

Model parameters

\begin{tabular}{lll}
\hline Parameter & Value & The definition \\
\hline$D$ & 2 & Particle search dimension \\
$x_{\max }$ & 1 & The max position of the particle search \\
$x_{\min }$ & 0 & The min position of the particle search \\
$v_{\min }$ & -1 & Min particle search speed \\
$v_{\max }$ & 1 & Max particle search speed \\
$N$ & 20 & Total population size \\
$m$ & 4 & Number of subgroups \\
$M 1$ & 5 & Number of particles per subgroup \\
$\omega_{\max }$ & 0.9 & Max inertia factor \\
$\omega_{\min }$ & 0.4 & Min inertia factor \\
$C 1$ & 1.4962 & Particle learning factor \\
$C 2$ & 1.4962 & Subgroup learning factor \\
$C 3$ & 1.4962 & Global learning factor \\
$T$ & 500 & Number of iterations \\
$T h$ & 3 & Environmental variable \\
\hline
\end{tabular}


According to the actual data of the study area, the dominant tree species in the plot is thick tree. Select the $D B H$ model of the thick tree to calculate the growth of the dominant tree species in each environment, and then calculate the environmental change threshold $\theta$. When $T h=1, \theta=1.6575$, and when $T h=2, \theta=3.3472$.

The results of the model output under the three environmental variables are shown in Fig.5, Fig.6, and Fig.7, representing the spatial distribution of the target tree in the stand when $T h=1, T h=2$ and $T h=3$, respectively.

Fig. 5(a), Fig. 6(a) and Fig. 7(a) indicates the spatial position of the target tree in the forest, the red dot indicates the forest to be adjusted (i.e., the target tree). The number in Fig. 5(b), Fig. 6(b) and Fig. 7(b) indicates the number of the target tree in the plot. According to the dynamic diameter of the target tree, such as $D B H$, tree height, crown width and height under the branch, the reason for adjusting the target tree can be obtained. For example, the target tree has too close neighboring wood or the health index is too low, and the competition index is too large.

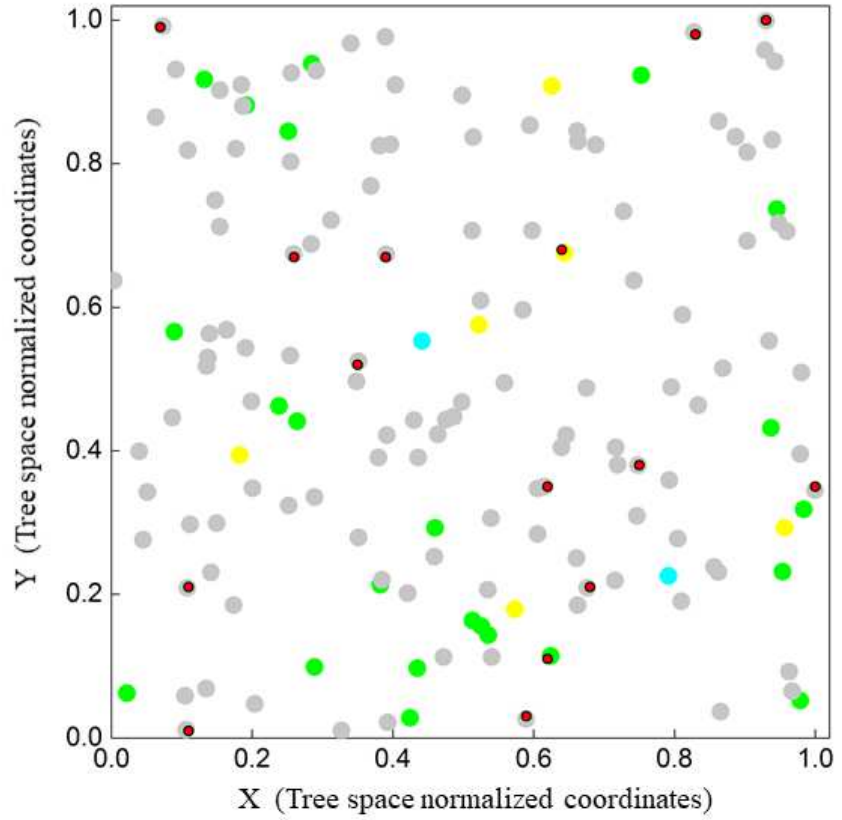

(a)

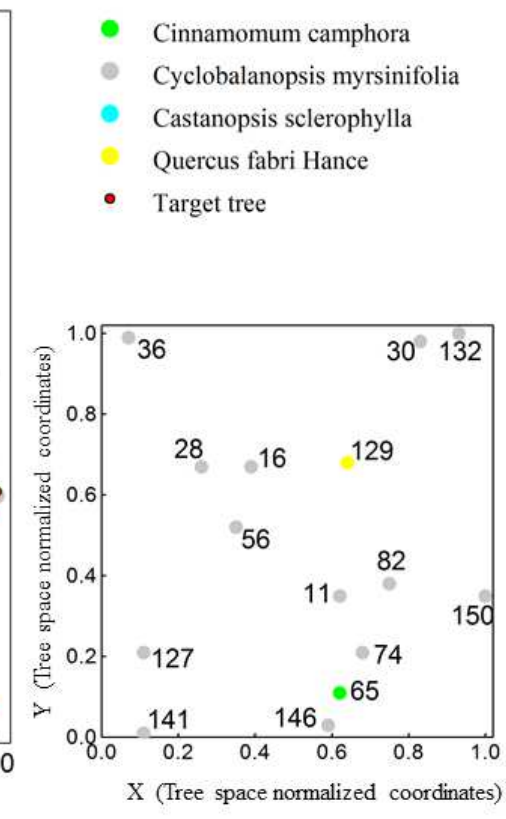

(b)

Fig. 5. The spatial distribution of the target trees in $T h=1$. (a) The spatial position of the target trees in the stand; (b) The number of the target trees. 


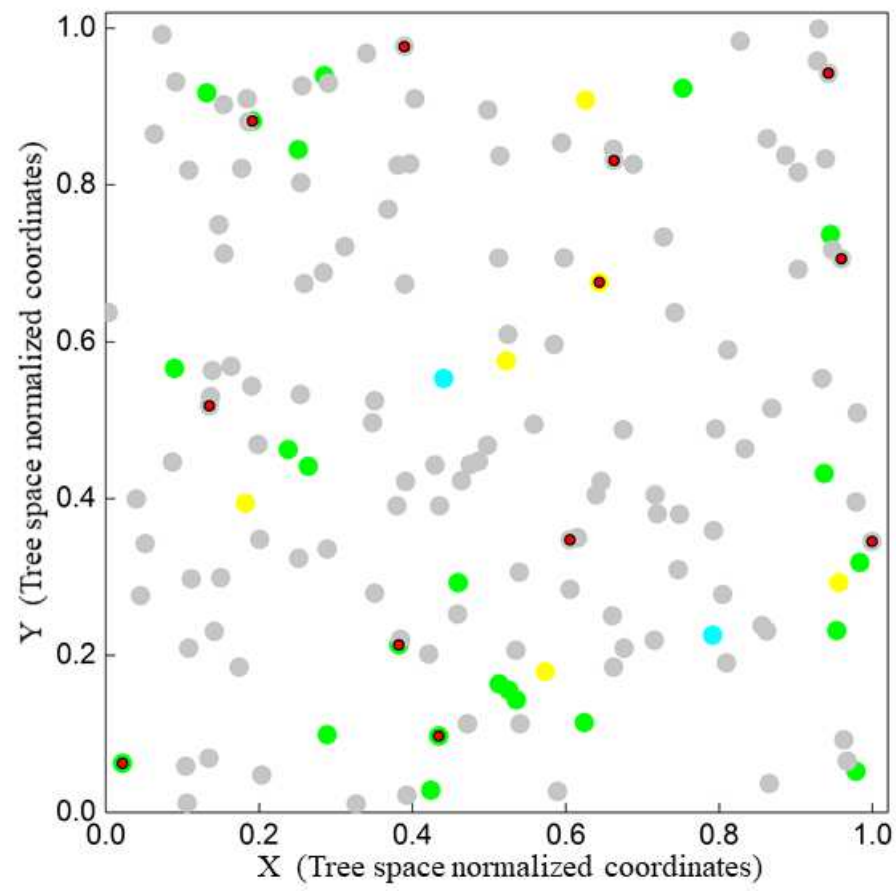

(a)

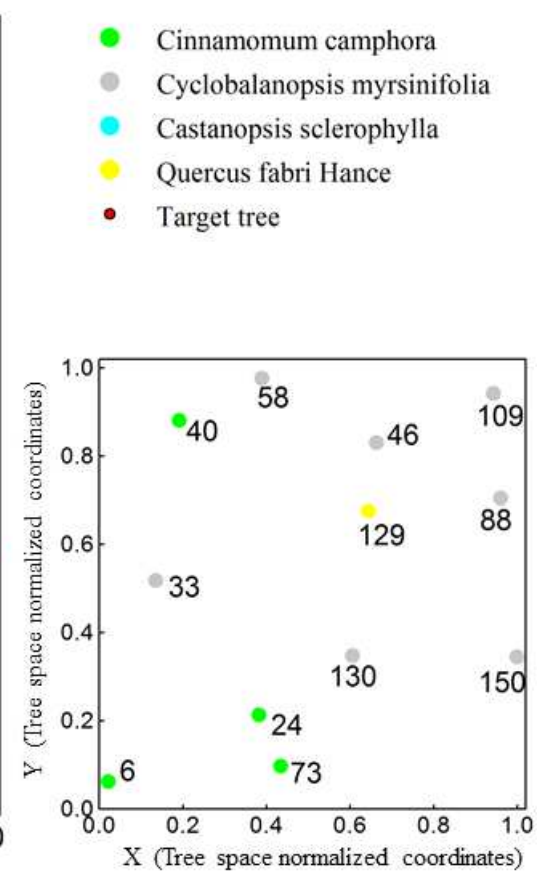

(b)

Fig. 6. The spatial distribution of the target trees in $T h=2$. (a) The spatial position of the target trees in the stand; (b) The number of the target trees.

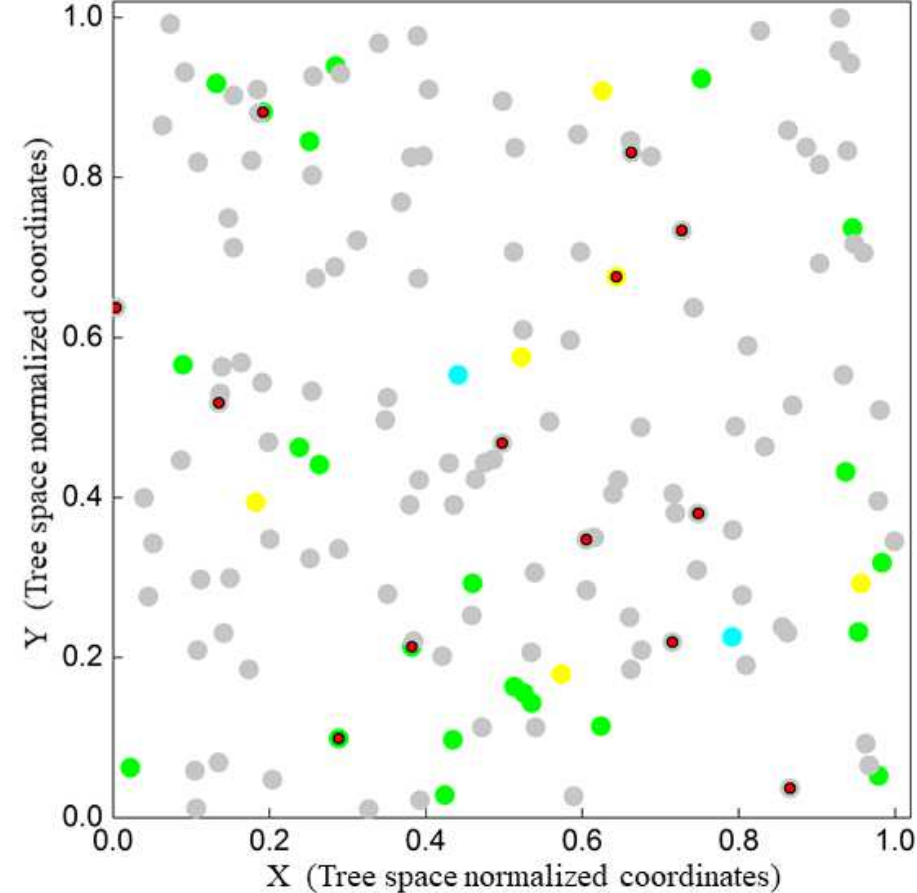

(a)

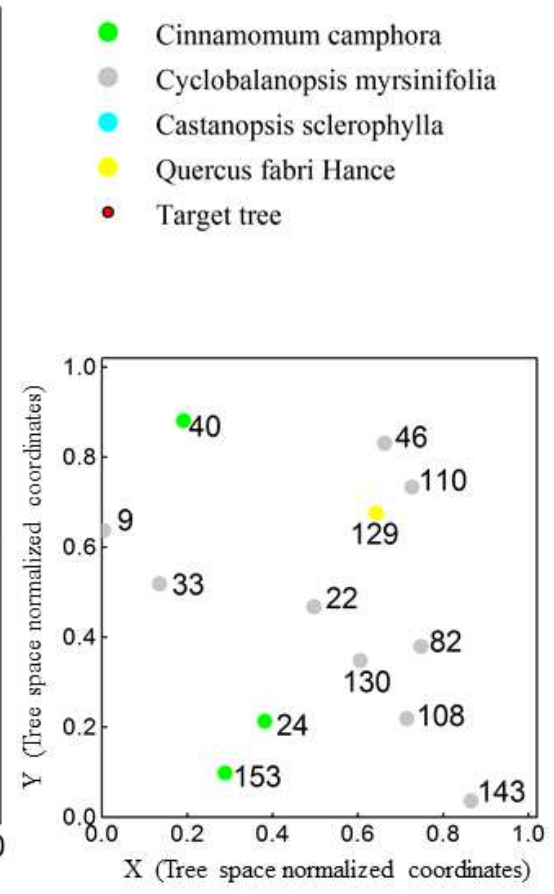

(b)

Fig. 7. The spatial distribution of the target trees in $T h=3$. (a) The spatial position of the target trees in the stand; (b) The number of the target trees. 


\subsubsection{Evaluation of the Optimization Effect of Forest Structure}

The average change of the indicators before and after the structural adjustment of the optimization model, is shown in Table 4. The first six items are the target tree structure indicators, and the last three items are the stand structure indicators. For the magnitude of the change of each indicator, a positive number indicates that the value of the indicator has increased from its original value, and a negative number indicates that the value of the indicator has reduced from its original value. It can be clearly seen from Table 4 that, after each adjustment of the stand structure, the mixed degree, fore st index, open ratio, health and stand homogeneous index are improved greatly compared with those without adjustment. The competition index and angle index have declined compared with those before adjustment. After each adjustment, the mingling intensity, volume, neighborhood comparison and open ratio have increased from their previous value. We can draw a conclusion that with the adjustment of the forest structure, the forest structure is getting better and better. Therefore, the forest spatial dynamic multiobjective optimization model constructed in this paper is reasonable and feasible.

\section{Table 4}

The average change of spatial and non-spatial structure indexes before and after regulation

\begin{tabular}{|c|c|c|c|c|c|c|c|}
\hline & & $\mathrm{Th}=1$ & & $\mathrm{Th}=2$ & & $T h=3$ & \\
\hline Parameter & $\begin{array}{l}\text { Before } \\
\text { regulatio } \\
n\end{array}$ & $\begin{array}{l}\text { After } \\
\text { regulatio } \\
\mathrm{n}\end{array}$ & $\begin{array}{l}\text { Trend } \\
\text { and } \\
\text { magnitud } \\
\text { e }(\%)\end{array}$ & $\begin{array}{l}\text { After } \\
\text { regulatio } \\
\mathrm{n}\end{array}$ & $\begin{array}{l}\text { Trend } \\
\text { and } \\
\text { magnitud } \\
\text { e (\%) }\end{array}$ & $\begin{array}{l}\text { After } \\
\text { regulation }\end{array}$ & $\begin{array}{l}\text { Tren } \\
\mathrm{d} \text { and } \\
\text { magn } \\
\text { itude } \\
(\%)\end{array}$ \\
\hline
\end{tabular}




\begin{tabular}{|c|c|c|c|c|c|c|c|}
\hline $\begin{array}{l}\text { Mingling } \\
\text { intensity }\end{array}$ & 0.429 & 0.561 & 30.8 & 0.618 & 44.1 & 0.603 & 40.6 \\
\hline $\begin{array}{l}\text { Competition } \\
\text { index }\end{array}$ & 0.363 & 0.2084 & -42.6 & 0.251 & -30.7 & 0.225 & -37.9 \\
\hline Angle index & 0.634 & 0.537 & -15.3 & 0.504 & -20.5 & 0.551 & -13.1 \\
\hline Volume & 0.485 & 0.513 & 5.8 & 0.508 & 4.7 & 0.497 & 2.5 \\
\hline Neighborhoo & & & & & & & \\
\hline $\begin{array}{l}\mathrm{d} \\
\text { comparison }\end{array}$ & 0.587 & 0.735 & 25.2 & 0.712 & 21.3 & 0.698 & 18.9 \\
\hline Open ratio & 0.619 & 0.713 & 15.2 & 0.71 & 14.7 & 0.692 & 11.8 \\
\hline
\end{tabular}

\section{Conclusions}

In this paper, taking the natural secondary forest of the state-owned forest farm in Longhushan, Dongting Lake, as an example, a proposed model is tested for actual forest stand. The adjustment of the forest space by the target tree of the model output can significantly improve the spatial structure of the forest stand. Based on the existing PSO algorithm, an environment detection mechanism is introduced to detect changes in the surrounding environment. The performance of the improved algorithm is tested using four test functions (i.e., FDA1, FDA3, dMOP1, dMOP3). Compared with the other two algorithms for solving dynamic multi-objective optimization problems (PDMIOA and dDMSMOPSO algorithm), the algorithm proposed possesses better performance in convergence, solution coverage, and tracking aspects. A dynamic multi-objective optimization model for the spatial structure of natural secondary forests is constructed by screening relevant parameters related to the stand structure, objective functions, and constraints. When evaluating the forest management model, the economic and social benefits can be maximized through the model evaluation. 
When solving the multi-objective optimization problem, it is necessary to further study the sensitivity to the environment and the reaction speed to enhance the adaptability of the multi-group particle swarm optimization algorithm. In fact, the superiority of this algorithm can be demonstrated in various fields. There are many types of intelligent algorithms, and they all have their own advantages and disadvantages. In future research, multi-population particle swarm optimization algorithms can be combined with other intelligent algorithms, such as genetic algorithm, ant colony algorithm, bee colony algorithm, etc. It is feasible to enable it to be applied to more fields and create greater practical value. Not only the growth of trees affects the structure of the forest, but also the climate change has a huge impact on it. Due to the lack of climate data, this study did not consider this aspect. Therefore, in the next step, we will continue to study the spatial structure optimization model of climate change so as to explore the impact of climate factors on forest spatial structure.

\section{Acknowledgements}

This research was supported by grants from the National Natural Science Foundation of China (No.31570627), Hunan Forestry Science and Technology Project (No.XLK201740), and Hunan Science and Technology Innovation Platform and Talent Plan (No.2017TP1022). 


\section{References}

[1] Hong Z. A New Method of Cooperative PSO: Multiple Particle Swarm Optimizers with Inertia Weight with Diversive Curiosity[M]// Intelligent Control and Innovative Computing. 2012.

[2] Coello C A C, Lamont G B, Veldhuizen D A V. Evolutionary Algorithms for Solving Multi-Objective Problems[J]. 2002.

[3] Abraham A, Das S, Roy S. Swarm Intelligence Algorithms for Data Clustering[J].

[4] Rajendran C, Ziegler H. Ant-colony algorithms for permutation flowshop scheduling to minimize makespan/total flowtime of jobs[J]. European Journal of Operational Research, 2007, 155(2):426-438.

[5] Neshat M, Sepidnam G, Sargolzaei M, et al. Artificial fish swarm algorithm: a survey of the state-of-the-art, hybridization, combinatorial and indicative applications[J]. Artificial Intelligence Review, 2014, 42(4):965-997.

[6] Berg V D, Ewout, friedlander, et al. Probing the pareto frontier for basis pursuit solutions[j]. Siam journal on Scientific Computing, 2008, 31(2):890-912.

[7] $\mathrm{Wu} \mathrm{Z}$, Zhou J. A self-adaptive particle swarm optimization algorithm with individual coefficients adjustment [C] // Computational Intelligence and Security, 2007 International Conference on. IEEE， 2007: 133-136.

[8] Urade H S , Patel R. Dynamic particle swarm optimization to solve multi- objective optimization problem [J]. Procedia Technology, 2012, 6: 283- 290.

[9] Hanson J J, Lorimer C G. Forest structure and light regimes following moderate wind storms: implications for multi - cohort management[j]. Ecological applications, 2007, 17(5):1325-1340. 
[10] Venema H D, Calamai P H, Fieguth P. Forest structure optimization using evolutionary programming and landscape ecology metrics $[\mathrm{J}]$. European Journal of Operational Research, 2005, 164(2):423-439.

[11] Zhao Q, Zheng G, Huang Q. Characteristics of Urban Forest Landscape Pattern and Optimization of Urban Forest Spatial Structure: A Case Study of Nanjing City[J]. Acta Geographica Sinica, 2007.

[12] Urade H S, Patel R. Dynamic Particle Swarm Optimization to Solve Multiobjective Optimization Problem[J]. Procedia Technology, 2012, 6(none).

[13] Eichfelder G. A Constraint Method in Nonlinear Multi-Objective Optimization[M]// Multiobjective Programming and Goal Programming. Springer Berlin Heidelberg, 2009.

[14] Martinez S Z, Montaño, A. A, Coello Coello C A. A nonlinear simplex search approach for multi-objective optimization[C]// Evolutionary Computation. IEEE, 2011. [15] Kint V, Lust N, Ferris R, et al. Quantification of forest stand structure applied to Scots pine (Pinus sylvestris L.) forests.[J]. Investigacion Agraria Sistemas Y Recursos Forestales, 2000, 10(1):147-164.

[16] Rothley, Kristina D. Use of multiobjective optimization models to examine behavioural trade-offs of white-tailed deer habitat use in forest harvesting experiments[J]. Canadian Journal of Forest Research, 2002, 32(7):1275-1284.

[17] Song C, Dickinson M B. Extracting forest canopy structure from spatial information of high resolution optical imagery: tree crown size versus leaf area index $[\mathrm{J}]$. International Journal of Remote Sensing, 2008, 29(19):5605-5622.

[18] Zhu W, He X, Chen W, et al. Quantitative analysis of urban forest structure: A case study on Shenyang arboretum[J]. Chinese Journal of Applied Ecology, 2003, 14(12):2090-2094. 
[19] Chen D K, Zhou X F, Zhao H X, et al. Study on the structure, function and succession of the four types in natural secondary forest[J]. Journal of Northeast Forestry University, 1982, 10(2):1-20.

[20] Ke L, Kwong S, Cao J, et al. Achieving balance between proximity and diversity in multi-objective evolutionary algorithm[J]. Information Sciences, 2012, 182(1):220242.

[21] Sahmoud S, Topcuoglu H R. A Memory-Based NSGA-II Algorithm for Dynamic Multi-objective Optimization Problems[M]// Applications of Evolutionary Computation. Springer International Publishing, 2016.

[22] Annauth R, Rughooputh H C S. Optimization of OFDM systems resources using multi-objective Estimation Distribution Algorithms[C]//Information and Communication Technologies (WICT), 2012 World Congress on. IEEE, 2012: 703-708. [23] Lappi J, Lempinen R. A linear programming algorithm and software for forestlevel planning problems including factories[J]. Scandinavian Journal of Forest Research, 2014, 29(sup1): 178-184.

[24] Esmin A A A, Coelho R A, Matwin S. A review on particle swarm optimization algorithm and its variants to clustering high-dimensional data[J]. Artificial Intelligence Review, 2015, 44(1):23-45.

[25] Coello C A C, Pulido G T, Lechuga M S. Handling multiple objectives with particle swarm optimization[J]. IEEE Transactions on Evolutionary Computation, 2004, $8(3): 256-279$.

[26] Hongbing D, Qingchun W, Qingli W, et al. On riparian forest buffers and riparian management[J]. Chinese Journal of Applied Ecology, 2001, 12:951-954. 
[27] $\mathrm{Wu} \mathrm{Z}$, Zhou J. A self-adaptive particle swarm optimization algorithm with individual coefficients adjustment[C]//Computational Intelligence and Security, 2007 International Conference on. IEEE, 2007: 133-136.

[28] Zhang G, Shao X, Li P, et al. An effective hybrid particle swarm optimization algorithm for multi-objective flexible job-shop scheduling problem[J]. Computers \& Industrial Engineering, 2009, 56(4): 1309-1318.

[29] Coello C A C, Pulido G T, Lechuga M S. Handling multiple objectives with particle swarm optimization[J]. IEEE Transactions on evolutionary computation, 2004, 8(3): 256-279.

[30] Wu Z, Zhou J. A self-adaptive particle swarm optimization algorithm with individual coefficients adjustment[C]//Computational Intelligence and Security, 2007 International Conference on. IEEE, 2007: 133-136.

[31] Suresh K, Ghosh S, Kundu D, et al. Inertia-adaptive particle swarm optimizer for improved global search[C]//Intelligent Systems Design and Applications, 2008. ISDA'08. Eighth International Conference on. IEEE, 2008, 2: 253-258.

[32] Zhang G, Shao X, Li P, et al. An effective hybrid particle swarm optimization algorithm for multi-objective flexible job-shop scheduling problem[J]. Computers \& Industrial Engineering, 2009, 56(4): 1309-1318.

[33] Zhang L. Evolutionary dynamic multi-objective optimization algorithm and its application[D]. Xidian University, 2014.

[34] Zhang W. Research and application of dynamic multi-objective optimization algorithm based on particle swarm optimization algorithm[D]. Zhengzhou University, 2013.

[35] Liu Y, Qin Z, Shi Z, Lu J. Center particle swarm optimization. Neurocomputing. $2007 ; 70: 672-9$. 
[36] Hakli H, Uguz H. A novel particle swarm optimization algorithm with Levy flight. Appl Soft Compute. 2014;23:333 - 45.

[37] Hemlata S. Urade, Rahila Patel,Dynamic Particle Swarm Optimization to Solve Multi-objective Optimization Problem,Procedia Technology, Volume 6,2012,Pages 283 290. 